\title{
ON THE COMPACTNESS OF THE EVOLUTION OPERATOR GENERATED BY CERTAIN NONLINEAR $\Omega$-ACCRETIVE OPERATORS IN GENERAL BANACH SPACES
}

\author{
ATHANASSIOS G. KARTSATOS
}

(Communicated by Barbara Lee Keyfitz)

\begin{abstract}
A sufficient condition is given for the compactness of the evolution operator $U(t, s)$ generated by a family of nonlinear $\omega$-accretive operators $A(t)$. This family $A(t)$ satisfies a time-dependence condition which is not covered by the results of Calvert and the author. It is also shown that the main part of this sufficient condition is necessary.
\end{abstract}

\section{INTRODUCTION AND PRELIMINARIES}

In what follows, $\mathscr{R}, \mathscr{R}_{+}$are the real line and the set $[0, \infty)$, respectively. Also, $T$ is a fixed positive number and $X$ is a real Banach space.

Definition 1. We denote by $\mathscr{F}$ the set of all integrable functions $f:[0, T] \times$ $[0, T] \rightarrow \mathscr{R}_{+}$such that:

(i) $f(s, s)=0, f(s, t)=f(t, s), f(t, s) \leq f(t, r)+f(s, r), 0 \leq r \leq s \leq$ $t<T$;

(ii) for each $s \in[0, T)$, we have $f(\cdot, s) \in \mathscr{L}^{1}(0, T)$ and $f(\cdot+s, \cdot) \in$ $\mathscr{L}^{1}(0, T-s)$;

(iii) for each $h \in(0, T)$, the function

$$
F(s, r) \equiv \int_{0}^{h} f(t+s, t+r) d t
$$

is upper semicontinuous on $[0, T-h] \times[0, T-h]$, i.e., $\left\{s_{n}\right\},\left\{r_{n}\right\} \subset[0, T-$ $h] \times[0, T-h]$ and $s_{n} \rightarrow s_{0}, r_{n} \rightarrow r_{0}$ imply that

$$
\limsup _{n \rightarrow \infty} F\left(s_{n}, r_{n}\right) \leq F\left(s_{0}, r_{0}\right) \text {. }
$$

Definition 2. Let $\mathscr{D} \subset[0, T) \times X, \mathscr{D}(s)=\{x \in X:(s, x) \in \mathscr{D}\}, s \in[0, T)$. Let $U(t, s), 0 \leq s \leq t<T$, be a family of operators in $X$. Then $U(t, s)$

Received by the editors April 28, 1993 and, in revised form, October 13, 1993.

1991 Mathematics Subject Classification. Primary 47H20; Secondary 39B70, 47H06.

Key words and phrases. Compact evolution operator, $\omega$-accretive operator, compact resolvents, Crandall-Pazy theory. 
is said to be an evolution operator of type $\mathscr{E}(\mathscr{D}, \omega, f)$, where $\omega \in \mathscr{R}$ and $f \in \mathscr{F}$, provided that:

(E1) if $0 \leq r \leq s \leq t<T, U(t, s)$ maps $\mathscr{D}(s)$ into $\mathscr{D}(t), U(s, s)$ is the identity on $\mathscr{D}(s)$ and $U(t, s) U(s, r)=U(t, r)$ on $\mathscr{D}(r)$;

(E2) for $s \in[0, T)$ and $x \in \mathscr{D}(s), U(\cdot, s) x \in C([s, T), X)$;

(E3) for $0 \leq r \leq s<T, 0 \leq t<T-s, x \in \mathscr{D}(s)$ and $y \in \mathscr{D}(r)$,

$$
\|U(t+s, s) x-U(t+r, r) y\| \leq e^{\omega t}\|x-y\|+\int_{0}^{t} e^{\omega(t-\tau)} f(\tau+s, \tau+r) d \tau .
$$

Definition 3. For $x, y \in X$ we set

$$
(x, y)_{+} \equiv \inf _{\lambda>0} \lambda^{-1}(\|x+\lambda y\|-\|x\|) .
$$

An operator $A: X \rightarrow 2^{X}$ is $\omega$-accretive, where $\omega \in \mathscr{R}$, whenever

$$
(x-y, u-v)_{+} \geq-\omega\|x-y\| \text { for all } x, y \in D(A), u \in A x, v \in A y .
$$

Condition I. Let $A(t), t \in[0, T)$, be a family of $\omega$-accretive operators, for some $\omega \in \mathscr{R}_{+}$. Let $\mathscr{D}=\{(t, x) \in[0, T) \times X: x \in D(A(t))\}$. Let $f \in \mathscr{F}$ and let $U(t, s)$ be an evolution operator of type $\mathscr{E}(\mathscr{D}, \omega, f)$. Suppose that for $0 \leq s<t<T, x \in \mathscr{D}(s), 0 \leq r<T$ and $w \in A(r) z$ we have

$$
\begin{aligned}
& e^{-\omega t}\|U(t, s) x-z\|-e^{-\omega s}\|x-z\| \\
& \quad \leq \int_{s}^{t} e^{-\omega \tau}\left((U(\tau, s) x-z,-w)_{+}+f(\tau, r)\right) d \tau .
\end{aligned}
$$

Condition II. $R(I+\lambda A(t+\lambda)) \supset \overline{D(A(t))}$ for all $t \in[0, T)$ and all $\lambda \in(0, T-t)$ with $\lambda \omega<1$.

Calvert and the author gave in [4] a result, under Conditions I and II, containing necessary and sufficient conditions for the compactness of the operator $U(t, s)$. However, this result does not cover an important case of operators $A(t)$, i.e., operators satisfying the following condition.

Condition III. (i) There exists $\omega \in \mathscr{R}_{+}$such that for each $t \in[0, T]$ the operator $A(t)$ is $\omega$-accretive and $R(I+\lambda A(t))=X$ for $\lambda \in \Lambda \equiv\left(0, \lambda_{0}\right)$, where $\lambda_{0}$ is a fixed positive constant such that $\lambda_{0} \omega<1$.

(ii) There is a continuous function $h:[0, T] \rightarrow X$ of bounded variation on $[0, T]$, and an increasing function $L: \mathscr{R}_{+} \rightarrow \mathscr{R}_{+}$such that

$$
\left\|A_{\lambda}(t) x-A_{\lambda}(s) x\right\| \leq\|h(t)-h(s)\| L(\|x\|)\left(1+\left\|A_{\lambda}(s) x\right\|\right),
$$

for all $\lambda \in \Lambda, t, s \in[0, T]$, and $x \in X$, where

$$
J_{\lambda}(t)=(I+\lambda A(t))^{-1}, \quad A_{\lambda}(t)=\lambda^{-1}\left(I-J_{\lambda}(t)\right) .
$$

In fact, under Condition III, the integral inequality in Condition I will have the function $L(\|x\|)\|h(\tau)-h(r)\|(1+\|w\|)$ in place of the function $f(\tau, r)$. This can be seen in the paper of Tanaka [15, p. 51]. Because of the presence of the factor $L(\|x\|)(1+\|w\|)$, the function $f(\tau, r)$ does not satisfy (i) of Definition 1. Thus, the sufficiency part of the proof of the main result in [4] does not go through in the present setting, and some modifications are needed in the proof of the necessity part. 
Our purpose in this paper is to give a set of sufficient conditions for the compactness of the Crandall-Pazy evolution operator generated by a family of $\omega$-accretive operators satisfying Condition III. This is the content of Theorem 1. Theorem 2 shows that two of these conditions are also necessary for the compactness of this evolution operator.

We collect some properties of $\omega$-accretive operators $A$ and the directional derivative $(x, y)_{+}$. The books of Barbu [2] and Lakshmikantham and Leela [13] are excellent references on the subject of accretive operators. We assume that $R(A+\lambda I)=X$ for all $\lambda \in \Lambda$. We set $J_{\lambda}=(I+\lambda A)^{-1}, A_{\lambda}=(1 / \lambda)\left(I-J_{\lambda}\right)$ and always assume that $\lambda \in \Lambda$.

(i) $\left\|J_{\lambda} x-J_{\lambda} y\right\| \leq(1-\lambda \omega)^{-1}\|x-y\|, x, y \in X$.

(ii) $\left\|J_{\lambda} x-x\right\| \leq \lambda(1-\lambda \omega)^{-1}|A x|, \quad x \in D(A)$, where

$$
|A x|=\inf _{y \in A x}\{\|y\|\}, x \in D(A) .
$$

(iii) $A_{\lambda}$ is $\omega_{1}$-accretive with $\omega_{1}=\omega(1-\lambda \omega)^{-1}$. Also,

$$
\left\|A_{\lambda} x-A_{\lambda} y\right\| \leq \lambda^{-1}\left(1+(1-\lambda \omega)^{-1}\right)\|x-y\|, x, y \in X .
$$

(iv) $A_{\lambda} x \in A J_{\lambda} x, x \in X$.

For $x, y, z \in X$ and $\alpha, \beta \in \mathscr{R}$ we have

(1) $(x, \alpha x+y)_{+}=\alpha\|x\|+(x, y)_{+}$;

(2) $(\alpha x, \beta y)_{+}=|\beta|(x, y)_{+}$for $\alpha \beta>0$;

(3) $(x, y+z)_{+} \leq(x, y)_{+}+(x, z)_{+}$;

(4) $(x, y)_{+} \leq\|x+y\|-\|x\|$;

(5) $\left|(x, y)_{+}\right| \leq\|y\|$;

(6) the function $(x, y)_{+}: X \times X \rightarrow \mathscr{R}$ is upper semicontinuous.

We give below some of the properties of the Crandall-Pazy evolution operator $U(t, s)$. The rest of its properties can be found in [5]. We let $\Delta=\{(t, s) \in$ $\left.[0, T]^{2}: 0 \leq s \leq t \leq T\right\}$ and assume that Condition III is satisfied. We let $D=D(A(0))$. We know that $\overline{D(A(t))}=\bar{D}=$ const. for all $t \in[0, T]$.

(i) For each $(t, s) \in \Delta, \quad U(t, s): \bar{D} \rightarrow \bar{D}$ and the mapping $(t, s, x) \rightarrow$ $U(t, s) x$ is continuous on $\Delta \times \bar{D}$.

(ii) For every $r, s, t \in[0, T]$ with $r \leq s \leq t$, we have $U(t, s) U(s, r)=$ $U(t, r)$.

(iii) $\|U(t, s) x-U(t, s) y\| \leq e^{\omega(t-s)}\|x-y\|, \quad(t, s) \in \Delta, \quad x, y \in \bar{D}$.

\section{Main Results}

Our main result is contained in the following theorem.

Theorem 1. Assume that Condition III holds. Then the Crandall-Pazy evolution operator $U(t, s)$ is compact on $\bar{D}$, for each $t, s \in \Delta$ with $t>s$, if the following additional conditions are satisfied:

(i) For every $x \in \bar{D}, J_{\lambda}(t) x$ is continuously differentiable w.r.t. $t \in[0, T]$, for all $\lambda \in \Lambda$, with derivative $J_{\lambda}^{\prime}(t) x$ of bounded variation on $[0, T]$. In addition, for every bounded set $B \subset \bar{D}$,

$$
\lim _{\lambda \rightarrow 0} \lambda \int_{0}^{T}\left\|J_{\lambda}^{\prime}(t) x\right\| d t=0 \text { uniformly w.r.t. } x \in B .
$$


(ii) The resolvents $J_{\lambda}(t)$ are compact on $\bar{D}$ for each $t \in[0, T]$ and each $\lambda \in \Lambda$.

(iii) Given $\epsilon>0, s \in[0, T), t_{0} \in(s, T]$ and a bounded set $B \subset \bar{D}$, there exists $\delta=\delta\left(s, t_{0}, \epsilon, B\right)$ such that

$$
\left\|U\left(t_{0}, s\right) x-U(t, s) U\left(t_{0}, s\right) x\right\|<\epsilon
$$

for every $t \in(s, s+\delta)$ and every $x \in B$.

The following statement is important for the applicability of Theorem 1 in reflexive Banach spaces.

Remark 1. Let Condition III hold with $h(t) \equiv t$ and let $X$ be reflexive. Then, for every $x \in \bar{D}, \quad J_{\lambda}(t) x$ is absolutely continuous and differentiable a.e. w.r.t. $t \in[0, T]$ for all $\lambda \in \Lambda$. In addition, for every bounded set $B \subset \bar{D}$,

$$
\lim _{\lambda \rightarrow 0} \lambda \int_{0}^{T}\left\|J_{\lambda}^{\prime}(t) x\right\| d t=0 \text { uniformly w.r.t. } x \in B .
$$

Proof. We observe that $A_{\lambda}(t)=(1 / \lambda)\left(I-J_{\lambda}(t)\right)$ and

$$
\left\|J_{\lambda}(t) x-J_{\lambda}(s) x\right\| \leq \lambda L(\|x\|)|t-s|\left(1+\left\|A_{\lambda}(s) x\right\|\right) .
$$

We have

$$
\begin{aligned}
& \frac{\left\|J_{\lambda}(t) x-J_{\lambda}(s) x\right\|}{|t-s|} \leq \lambda L(\|x\|)\left[1+(1 / \lambda)\left\|\left(I-J_{\lambda}(t)\right) x\right\|\right] \\
& \leq L(\|x\|)\left(\lambda+\|x\|+\left\|J_{\lambda}(t) x\right\|\right) \\
&\left\|J_{\lambda}(t) x\right\| \leq\left\|J_{\lambda}(0) x\right\|+T L(\|x\|)\left(\lambda+\|x\|+\left\|J_{\lambda}(0) x\right\|\right)
\end{aligned}
$$

and

$$
\left\|J_{\lambda}(0) x\right\| \leq\left\|J_{\lambda}(0) x_{0}\right\|+\left(1-\lambda_{0} \omega\right)^{-1}\left\|x-x_{0}\right\|
$$

for any $x \in \bar{D}, \lambda \in \Lambda$, where $x_{0} \in \bar{D}$ is fixed. It is easy to see that (1)-(3) imply that $J_{\lambda}(t) x$ is Lipschitz-continuous, and thus absolutely continuous on $[0, T]$. Since $X$ is reflexive, $J_{\lambda}(t) x$ is differentiable a.e. w.r.t. $t \in[0, T]$ and the norm of the derivative, $\left\|J_{\lambda}^{\prime}(t) x\right\|$, is an integrable function on $[0, T]$ (cf. Brézis [3, Proposition A.1]). Moreover, from the above inequalities we also obtain that $\lambda\left\|J_{\lambda}^{\prime}(t) x\right\| \rightarrow 0$ as $\lambda \rightarrow 0$ uniformly for $(t, x) \in([0, T] \backslash M) \times B$, where $M$ is a measurable subset of $[0, T]$ with $\mu(M)=0$ and $B$ is a bounded subset of $\bar{D}$. This implies the validity of the integral condition.

Lemma 1. Let Condition III be satisfied and let $f:[0, T] \rightarrow X$ be a continuous function of bounded variation on $[0, T]$. Then, for any $s \in[0, T], x_{0} \in \bar{D}$, the Crandall-Pazy solution $x(t) \equiv U(t, s) x_{0}$ of the problem

$$
\begin{aligned}
x^{\prime}+A(t) x-f(t) & \ni 0, \quad t \in[s, T], \\
x(s) & =x_{0}
\end{aligned}
$$

is well defined. Moreover, the Crandall-Pazy solution $x_{\beta}(t)$ of the problem

$$
\begin{aligned}
x^{\prime}+A_{\beta}(t)-f(t) & =0, \quad t \in[s, T], \\
x(s) & =x_{0}
\end{aligned}
$$

is also well defined and $x_{\beta}(t) \rightarrow x(t)$ as $\beta \rightarrow 0^{+}$, uniformly on $[s, T]$. 
Proof. To show the existence of the solution $x(t)$ of $(\mathrm{C} 1)$, we observe first that the operator $A(t)-f(t)$ is $\omega$-accretive. We show that $R(I+\lambda(A(t)-f(t)))=X$ for all $\lambda \in \Lambda, t \in[0, T]$. Fix $\lambda \in \Lambda, t \in[0, T], y \in X$. Then there exists $x=$ $x(\lambda, t, y)$ such that $(I+\lambda A(t)) x \ni \lambda f(t)+y$. Thus, $(I+\lambda(A(t)-f(t))) x \ni y$. To show the $t$-dependence of $B(t) \equiv A(t)-f(t)$, we set $J_{\lambda}^{B}(t)=(I+\lambda B(t))^{-1}$. It is easy to see that $J_{\lambda}^{B}(t)=J_{\lambda}(t)(x+\lambda f(t)), \lambda \in \Lambda, t \in[0, T]$. Thus, as in Evans [6, proof of Theorem 2],

$$
\begin{aligned}
& \left\|J_{\lambda}^{B}(t) x-J_{\lambda}^{B}(s) x\right\| \\
& \quad \leq \lambda\|f(t)-f(s)\|+\lambda\|h(t)-h(s)\| L_{1}(\|x\|)\left(1+\left\|B_{\lambda}(s) x\right\|\right),
\end{aligned}
$$

where $L_{1}: \mathscr{R}_{+} \rightarrow \mathscr{R}_{+}$is an increasing function. Letting $g(t) \equiv \operatorname{Var}([0, t] ; f)+$ $\operatorname{Var}([0, t] ; h)$, where $\operatorname{Var}([0, t] ; f)$ denotes the total variation of $f$ on $[0, t]$, we obtain

$$
\left\|J_{\lambda}^{B}(t) x-J_{\lambda}^{B}(s) x\right\| \leq \lambda\left\|g(t) u_{0}-g(s) u_{0}\right\| L_{2}(\|x\|)\left(1+\left\|B_{\lambda}(s) x\right\|\right)
$$

for some new increasing function $L_{2}: \mathscr{R}_{+} \rightarrow \mathscr{R}_{+}$and any $u_{0} \in X$ with $\left\|u_{0}\right\|=$ 1 , i.e., a type of $t$-dependence exactly like the one in Condition (C). Thus, the solution $x(t) \equiv U(t, s) x_{0}$ exists by [5, Theorem 2.1].

Concerning Equation (C2), we set $D_{\beta}(t)=A_{\beta}(t)-f(t)$. We note that $A_{\beta}(t)$ is $\omega_{1}$-accretive, with $\omega_{1}=\omega /(1-\beta \omega)$, for all $\beta \in \Lambda$ and all $t \in[0, T]$. We now show that $R\left(I+\lambda D_{\beta}(t)\right)=X$ for all $\beta \in \Lambda, \lambda>0$ with $\lambda \omega_{1}<1$, and $t \in[0, T]$. Actually, it suffices to show that $R\left(I+\lambda A_{\beta}(t)\right)=X$ for the same values of $\beta, \lambda$ and $t$. To see this, fix $y \in X$ and consider the equation $\left(I+\lambda A_{\beta}(t)\right) x=y$. The solvability of this equation is equivalent to the existence of a fixed point for the mapping $\psi(x)$, where

$$
\psi(x) \equiv \frac{\beta}{\lambda+\beta}+\frac{\lambda}{\lambda+\beta} J_{\beta}(t) x .
$$

This mapping has a unique fixed point for the above values of the parameters $\beta, \lambda$ and $t$ (cf. [5, proof of Lemma 4.1]). We need to show that $D_{\beta}(t)$ satisfies a condition like $(\mathrm{C})$, but this follows immediately from the fact that the operator $A_{\beta}(t)$ satisfies such a condition (cf. [5, proof of Lemma 4.1]) and an inequality like (4).

In order to apply Lemma 4.1 of [5], it remains to show that

$$
J_{\lambda}^{D, \beta}(t) x \rightarrow J_{\lambda}^{B}(t) x \text { as } \beta \rightarrow 0^{+},
$$

for each $\lambda \in \Lambda, t \in[0, T]$, where $J_{\lambda}^{D, \beta}(t) \equiv\left(I+\lambda D_{\beta}(t)\right)^{-1}$. We set $J_{\lambda}^{\beta}(t)=$ $\left(I+\lambda A_{\beta}(t)\right)^{-1}$ and note that $J_{\lambda}^{D, \beta}(t) x=J_{\lambda}^{\beta}(x+\lambda f(t))$ and $J_{\lambda}^{B}(t) x=$ $J_{\lambda}(x+\lambda f(t))$. We also know that $J_{\lambda}^{\beta}(x+\lambda f(t)) \rightarrow J_{\lambda}(x+\lambda f(t))$ as $\beta \rightarrow$ $0^{+}$, for all $\lambda \in \Lambda, t \in[0, T]$, because of the continuity of the mapping $J_{q}(t): X \rightarrow D(A(t))$ in the variable $q$. This is shown, with $x+\lambda f(t)$ replaced by $x$, in [5, proof of Lemma 4.2]. Thus, $x_{\beta}(t) \rightarrow x(t)$ as $\beta \rightarrow 0^{+}$, uniformly on $[s, T]$.

Proof of Theorem 1. We consider the approximating problems

$$
\begin{aligned}
u^{\prime}+A_{\beta}(t) u & =0, \quad t \in[s, T], \\
u(s) & =x,
\end{aligned}
$$


and

$\left(\mathrm{F}_{\beta}\right)$

$$
\begin{aligned}
u^{\prime}+A_{\beta}(t) u & =J_{\lambda}^{\prime}(t) x+A_{\lambda}(t) x, \quad t \in[s, T], \\
u(s) & =J_{\lambda}(s) x,
\end{aligned}
$$

for fixed $\lambda \in \Lambda$ and $x \in \bar{D}$, where $\beta$ is a sufficiently small positive constant and $\omega_{1}$ is as in the proof of Lemma 1 . We let $u_{\beta}, \bar{u}_{\beta}$ denote the $C^{1}$ solutions of these problems, respectively. Since the functions $u_{\beta}, \bar{u}_{\beta}$ are continuously differentiable, we have that the function $\left\|\bar{u}_{\beta}(t)-u_{\beta}(t)\right\|$ is differentiable a.e. on $[s, T]$ and such that

$$
\begin{aligned}
(d / d t) & \left\|\bar{u}_{\beta}(t)-u_{\beta}(t)\right\|=\left(\bar{u}_{\beta}(t)-u_{\beta}(t), \bar{u}_{\beta}^{\prime}(t)-u_{\beta}^{\prime}(t)\right)_{+} \\
= & \left(\bar{u}_{\beta}(t)-u_{\beta}(t),-\left(A_{\beta}(t) \bar{u}_{\beta}(t)-A_{\beta}(t) u_{\beta}(t)\right)+J_{\lambda}^{\prime}(t) x+A_{\lambda}(t) x\right)_{+} \\
\leq & \left(\bar{u}_{\beta}(t)-u_{\beta}(t),-\left(A_{\beta}(t) \bar{u}_{\beta}(t)-A_{\beta}(t) u_{\beta}(t)\right)\right)_{+} \\
& +\left(\bar{u}_{\beta}(t)-u_{\beta}(t), J_{\lambda}^{\prime}(t) x+A_{\lambda}(t) x\right)_{+} \\
\leq & \left(\bar{u}_{\beta}(t)-u_{\beta}(t), J_{\lambda}^{\prime}(t) x+A_{\lambda}(t) x\right)_{+}+\omega_{1}\left\|\bar{u}_{\beta}(t)-u_{\beta}(t)\right\|,
\end{aligned}
$$

where we have used the $\omega_{1}$-accretiveness of the operators $A_{\beta}(t)$. Integrating this inequality $\left((x, y)_{+}\right.$is upper semicontinuous), we obtain

$$
\begin{aligned}
\| u_{\beta}(t) & -\bar{u}_{\beta}(t) \| \\
\leq & \left\|u_{\beta}(s)-\bar{u}_{\beta}(s)\right\|+\int_{s}^{t}\left(\bar{u}_{\beta}(\tau)-u_{\beta}(\tau), J_{\lambda}^{\prime}(\tau)+A_{\lambda}(\tau) x\right)_{+} d \tau \\
& +\omega_{1} \int_{s}^{t}\left\|\bar{u}_{\beta}(\tau)-u_{\beta}(\tau)\right\| d \tau .
\end{aligned}
$$

Working as in Remark 2.1 of [4], we see that this inequality implies

$$
\begin{aligned}
& \left\|\bar{u}_{\beta}(t)-u_{\beta}(t)\right\| \\
& \leq e^{\omega_{1}(t-s)}\left\|\bar{u}_{\beta}(s)-u_{\beta}(s)\right\|+\int_{s}^{t} e^{\omega_{1}(t-\tau)}\left(\bar{u}_{\beta}(\tau)-u_{\beta}(\tau), J_{\lambda}^{\prime}(\tau) x+A_{\lambda}(\tau)\right)_{+} d \tau .
\end{aligned}
$$

At this point we need to note that $u_{\beta}(t)=U_{\beta}(t, s) x, \bar{u}_{\beta}(t)=\bar{U}_{\beta}(t, s) J_{\lambda}(s) x$, where $U_{\beta}, \bar{U}_{\beta}$ are the Crandall-Pazy evolution operators associated with $\left(\mathrm{E}_{\beta}\right)$, $\left(\mathrm{F}_{\beta}\right)$, respectively. From Lemma 1 we have that $U_{\beta}(t, s) x \rightarrow U(t, s) x$ and $\bar{U}_{\beta}(t, s) J_{\lambda}(s) x \rightarrow J_{\lambda}(t) x$, as $\beta \rightarrow 0$, because $U(t, s) x$ is the Crandall-Pazy solution of the problem

$$
\begin{aligned}
u^{\prime}+A(t) u & \ni 0, \quad t \in[s, T], \\
u(s) & =x,
\end{aligned}
$$

and $J_{\lambda}(t) x$ is the Crandall-Pazy solution of the problem

$$
\begin{aligned}
u^{\prime}+A(t) u & \ni J_{\lambda}^{\prime}(t) x+A_{\lambda}(t) x, \quad t \in[s, T], \\
u(s) & =J_{\lambda}(s) x
\end{aligned}
$$

(cf. [5, Theorem 3.1]). Thus, for every $\lambda \in \Lambda$, every $x \in \bar{D}$ and every $t \geq s$, we have

(5)

$$
\begin{aligned}
\left\|J_{\lambda}(t) x-U(t, s) x\right\| \leq & e^{\omega(t-s)}\left\|J_{\lambda}(t) x-x\right\| \\
& +\int_{s}^{t} e^{\omega(t-u)}\left(J_{\lambda}(u) x-U(u, s) x, J_{\lambda}^{\prime}(u) x+A_{\lambda}(u) x\right)_{+} d u .
\end{aligned}
$$


We now use $A_{\lambda}(t) x \equiv(1 / \lambda)\left(I-J_{\lambda}(t) x\right)$ and the properties of $(x, y)_{+}$to obtain

$$
\begin{aligned}
&\left(J_{\lambda}(u) x-U(u, s) x, J_{\lambda}^{\prime}(u) x+A_{\lambda}(u) x\right)_{+} \\
&=(1 / \lambda)\left(J_{\lambda}(u) x-U(u, s) x, \lambda J_{\lambda}^{\prime}(u) x+\lambda A_{\lambda}(u) x\right)_{+} \\
& \leq(1 / \lambda)\left\|J_{\lambda}(u) x-U(u, s) x+\lambda J_{\lambda}^{\prime}(u) x+\lambda A_{\lambda}(u) x\right\| \\
&-(1 / \lambda)\left\|J_{\lambda}(u) x-U(u, s) x\right\| \\
&=(1 / \lambda)\left\|x-U(u, s) x+\lambda J_{\lambda}^{\prime}(u) x\right\|-(1 / \lambda)\left\|J_{\lambda}(u) x-U(u, s) x\right\| .
\end{aligned}
$$

Using

$$
\begin{aligned}
-e^{\omega(t-u)}\left\|J_{\lambda}(u) x-U(u, s) x\right\| & \leq-\left\|J_{\lambda}(u) x-U(u, s) x\right\| \\
& \leq\|x-U(u, s) x\|-\left\|x-J_{\lambda}(u) x\right\|,
\end{aligned}
$$

$t \geq u \geq s$, in (6), and then modifying (5) accordingly, we obtain

$$
\begin{aligned}
& (1 / \lambda) \int_{s}^{t}\left\|J_{\lambda}(u) x-x\right\| d u \leq e^{\omega(t-s)}\left\|J_{\lambda}(s) x-x\right\| \\
& \quad+(1 / \lambda) \int_{s}^{t} e^{\omega(t-u)}\left(2\|x-U(u, s) x\|+\lambda\left\|J_{\lambda}^{\prime}(u) x\right\|\right) d u
\end{aligned}
$$

for every $\lambda \in \Lambda$, every $t \in[s, T]$ and every $x \in \bar{D}$. Thus, if we replace $x$ in (7) by $U\left(t_{0}, s\right) x$, for some $t_{0} \in(s, T]$, we find

(8)

$$
\begin{aligned}
& \left\|\int_{s}^{t}\left(J_{\lambda}(u) U\left(t_{0}, s\right) x-U\left(t_{0}, s\right) x\right) d u\right\| \\
& \leq \int_{s}^{t}\left\|J_{\lambda}(u) U\left(t_{0}, s\right) x-U\left(t_{0}, s\right) x\right\| d u \\
& \leq \lambda e^{\omega(t-s)}\left\|J_{\lambda}(s) U\left(t_{0}, s\right) x-U\left(t_{0}, s\right) x\right\| \\
& \quad+\int_{s}^{t} e^{\omega(t-u)}\left(2\left\|U\left(t_{0}, s\right) x-U(u, s) U\left(t_{0}, s\right) x\right\|+\lambda\left\|J_{\lambda}^{\prime}(u) U\left(t_{0}, s\right) x\right\|\right) d u
\end{aligned}
$$

We observe that our hypotheses and (8) imply that for every $\epsilon>0, s \in$ $[0, T), t_{0} \in(s, T]$ and a bounded set $B \subset \bar{D}$ there exists $\delta=\delta\left(s, t_{0}, \epsilon, B\right)>$ 0 and $\lambda_{0}=\lambda_{0}\left(s, t_{0}, \epsilon, B\right)>0$ such that

$$
\left\|\int_{s}^{t}\left(J_{\lambda}(u) U\left(t_{0}, s\right) x-U\left(t_{0}, s\right) x\right) d u\right\|<\epsilon
$$

for every $t \in(s, s+\delta), \lambda \in\left(0, \lambda_{0}\right)$.

We fix $s, t$ and $t_{0}$ as above and consider the operator

$$
F(x) \equiv \int_{s}^{t} J_{\lambda}(u) U\left(t_{0}, s\right) x d u
$$

To show that the set $F(B)$ is relatively compact, we show first that the set $\mathscr{G}$ of functions

$$
f_{x}: u \rightarrow J_{\lambda}(u) U\left(t_{0}, s\right) x, \quad u \in[s, t], x \in B,
$$

is relatively compact. To this end, we must show that $\mathscr{G}$ is bounded, equicontinuous and such that each set

$$
Q_{u} \equiv\left\{f_{x}(u): f_{x} \in \mathscr{G}\right\}, \quad u \in[s, t]
$$


is relatively compact in $X$. All three properties follow from our considerations above as well as our hypotheses. In fact, the boundedness of $\mathscr{G}$ follows from (2) and (3) and its equicontinuity from the global Lipschitz-continuity of $J_{\lambda}(u) x$. The relative compactness of the set $Q_{u}$ in (10) is a consequence of the assumed compactness of the operator $J_{\lambda}(u)$. Thus, the set $\mathscr{G}$ is relatively compact. Let us pick a sequence $\left\{x_{n}\right\} \subset B$. Then, by the relative compactness of the set $\mathscr{G}$, there exists a subsequence $\left\{x_{n_{k}}\right\}$ such that the sequence of functions $f_{x_{n_{k}}}$ converges strongly and uniformly to some continuous function $f$ on $[s, t]$. Thus,

$$
\int_{s}^{t} J_{\lambda}(u) U\left(t_{0}, s\right) x_{n_{k}} d u \rightarrow \int_{s}^{t} f(u) d u
$$

This implies that the set

$$
F(B)=\left\{y \in X: y=\int_{s}^{t} J_{\lambda}(u) U\left(t_{0}, s\right) x d u, \text { for some } x \in B\right\}
$$

is relatively compact. We have shown that the operator $F$ maps bounded subsets $B$ of $\bar{D}$ onto relatively compact sets. Since $F$ is also continuous, it is compact.

Now, let $B$ be a bounded subset of $\bar{D}, \epsilon>0, s \in[0, T), t \in(s, T], t_{0} \in$ $(s, T]$ and $\epsilon_{0}=(t-s) \epsilon / 2$. Then there exist $\delta=\delta\left(s, t_{0}, \epsilon_{0}, B\right)>0, \lambda_{0}=$ $\lambda_{0}\left(s, t_{0}, \epsilon_{0}, B\right)>0$ so that (9) holds with $\epsilon_{0}$ instead of $\epsilon$ and such that the set $F(B)$ can be covered by a finite number of open balls of radius $\epsilon_{0}$. From (9) we see now that the set

$$
\left\{y: y=\int_{s}^{t} U\left(t_{0}, s\right) x d u=(t-s) U\left(t_{0}, s\right) x, \text { for some } x \in B\right\}
$$

can be covered by a finite number of open balls of radius $2 \epsilon_{0}=(t-s) \epsilon$. This implies that $U\left(t_{0}, s\right) B$ can be covered by a finite number of open balls of radius $\epsilon$ and proves the compactness of the operator $U\left(t_{0}, s\right)$ for any $s \in[0, T)$ and any $t_{0} \in(s, T]$. 1.

The next result shows the necessity of Conditions (ii) and (iii) of Theorem

Theorem 2. Assume that Condition III holds. Then if $U(t, s)$ is compact for all $(t, s) \in \Delta$ with $t>s$, Conditions (ii) and (iii) of Theorem 1 are true.

Proof. We give the complete proof although part of it follows as in [4]. Assume that $U(t, s)$ is compact for $(t, s) \in \Delta$ with $t>s$. Fix such a point $(t, s)$. To show that $J_{\lambda}(s) x$ is compact for every $\lambda \in \Lambda$, we note, as in Theorem 4 of Tanaka [15], that we have

$$
\begin{aligned}
\|U(t, s) x-z\| \leq e^{\omega(t-s)}\|x-z\| & \\
+\int_{s}^{t} e^{\omega(t-\tau)} & {\left[(U(\tau, s) x-z,-y)_{+}\right.} \\
& +L(\|x\|)\|h(\tau)-h(r)\|(1+\|y\|)] d \tau,
\end{aligned}
$$

for any $r \in[0, T], z \in D(A(r)), y \in A(r) z$ and any $x \in \bar{D}$. Letting $r=s$ 
and replacing $x$ and $z$ by $J_{\lambda}(s) x$, we have

$$
\begin{aligned}
& \left\|U(t, s) J_{\lambda}(s) x-J_{\lambda}(s) x\right\| \\
& \leq \int_{s}^{t} e^{\omega(t-\tau)}\left[\left(U(\tau, s) J_{\lambda}(s) x-J_{\lambda}(s) x,-y\right)_{+}\right. \\
& \left.\quad+L\left(\left\|J_{\lambda}(s) x\right\|\right) \int_{s}^{t}\|h(\tau)-h(s)\|\left(1+\left\|A_{\lambda}(s) x\right\|\right)\right] d \tau \\
& \leq \phi(\omega, t, s)\left\|A_{\lambda}(s) x\right\| \\
& \quad+e^{\omega(t-s)} L\left(\left\|J_{\lambda}(s) x\right\|\right)\left(1+\left\|A_{\lambda}(s) x\right\|\right) \int_{s}^{t}\|h(\tau)-h(s)\| d \tau
\end{aligned}
$$

because $A_{\lambda}(s) x \in A(s) J_{\lambda}(s) x$. Here, $\phi(\omega, t, s)=\omega^{-1}\left(e^{\omega(t-s)}-1\right)$ if $\omega>0$ and $(t-s)$ if $\omega=0$. Let $B \subset \bar{D}$ be bounded. Then $J_{\lambda}(s) B$ and $A_{\lambda}(s) B$ are uniformly bounded ( $\lambda$ is fixed here). Since $L(\|x\|)$ is also bounded on $B$, we have that, given $\epsilon>0$, there exists $\delta=\delta(s, \lambda, \epsilon, B)>0$ such that

$$
\left\|U(t, s) J_{\lambda}(s) x-J_{\lambda}(s) x\right\|<\epsilon / 2
$$

for all $t \in(s, s+\delta)$. Since $J_{\lambda}(s) x$ is bounded and $U(t, s)$ is compact, the set $U(t, s) J_{\lambda}(s) B$ is relatively compact. As such, it can be covered by a finite number of open balls of radius $\epsilon / 2$. This last inequality implies that $J_{\lambda}(s) B$ can also be covered with a finite number of open balls of radius $\epsilon$. Thus, $J_{\lambda}(s)$ is compact for every $s \in[0, T)$. Its compactness for $s=T$ follows easily from its Lipschitz continuity on $[0, T]$.

To show (iii) of Theorem 1, we fix $\left(t_{0}, s\right) \in \Delta$ with $t_{0}>s$ and let $B \subset \bar{D}$ be bounded. Since the function $g:(t, x) \rightarrow U(t, s) x$ is continuous on $[s, T] \times \bar{D}$ and $Q \equiv[s, T] \times \overline{U\left(t_{0}, s\right) B}$ is a compact set, it follows that $g$ is uniformly continuous on $Q$. Thus, given $\epsilon>0$, there exists $\delta=\delta\left(s, t_{0}, \epsilon, B\right)>0$ such that

$$
\left\|U\left(t_{0}, s\right) x-U(t, s) U\left(t_{0}, s\right) x\right\|<\epsilon
$$

for all $t \in(s, s+\delta)$ and all $x \in B$. This completes the proof.

\section{Discussion}

It would certainly be interesting to know whether Theorem 1 is true without the assumption (i).

The author gave a result in [9] which contains the generation of an evolution operator for the functional evolution

$$
\begin{aligned}
x^{\prime}+A(t) x & =G\left(t, x_{t}\right), \quad t \in[0, T], \\
x_{0} & =\phi,
\end{aligned}
$$

where $A(t)$ is $m$-accretive for all $t \in[0, T]$ and satisfies Condition $(\mathrm{C})$ with $h(t) \equiv t$. The function $G$ is Lipschitzian in both variables and the function $\phi$ : $[-r, 0] \rightarrow X$ is also Lipschitz-continuous, where $r$ is a positive constant. The evolution operator $U(t, s)$ for (FDE) is defined on $\bar{D}=\overline{D(A(t))}=$ const and produces a solution $x(t), t \in[-r, T]$, which lies in the generalized domain $\hat{D}$, defined by

$$
\hat{D}=\hat{D}(A(0)) \equiv \hat{D}(A(t))=\left\{x \in X: \lim _{\lambda \rightarrow 0^{+}}\left\|A_{\lambda}(t) x\right\|<+\infty\right\},
$$


only on an initial interval $\left[0, T_{1}\right], T_{1} \leq T$, where it is actually Lipschitzcontinuous. This solution is the uniform limit of solutions $y^{n}(t)$ of the problems

$$
\begin{aligned}
y^{\prime}+A(t) y & =G\left(t, y_{t}^{n-1}\right), \quad t \in[0, T], \\
x(0) & =\phi(0) \in \hat{D},
\end{aligned}
$$

such that $y^{n}(t)=\phi(t), t \in[-r, 0]$. Such an evolution operator, with solution lying in $\hat{D}$ whenever $\phi(0) \in \hat{D}$, was also obtained by the author and Parrott in [11] (via a fixed-point theorem) and by Tanaka in [15], [16]. Tanaka's operator in [15] is actually more general than that of [9] and [11].

Shin and the author [12] gave some existence results by using the Schauder fixed-point theorem on such functional time-dependent problems involving compact evolution operators. The cases covered in that paper do not include the case of an evolution operator satisfying Condition III. Thus, another open problem arises in this direction. For conditions like the ones considered in [12], the reader is also referred to Pavel's book [14]. For evolutions with constant operators $A$, we cite the papers of Gutman [7], [8] and the book of Vrabie [17]. The original results involving the compactness of the operator $f \rightarrow x_{f}$ for nonlinear problems

$$
x^{\prime}+A x \ni f
$$

are due to Baras [1].

The author has given in [10] a method by which one can obtain large classes of compact evolution operators which are generated, for example, by $m$-accretive perturbations $A+B(t)$ of $m$-accretive compact semigroup generators $A$. These $m$-accretive operators $A+B(t)$ satisfy Condition III and Items (ii) and (iii) of Theorem 1.

\section{REFERENCES}

1. P. Baras, Compacité de l'opérateur $f \rightarrow u$ solution d'une équation non linéaire $(d u / d t)+$ $A u \ni f$, C. R. Acad. Sci. Paris 286 (1978), 1113-1116.

2. V. Barbu, Nonlinear semigroups and differential equations in Banach spaces, Noordhoff, Leyden, The Netherlands, 1975.

3. H. Brézis, Opérateurs maximaux monotones et semi-groupes de contractions dans les espaces de Hilbert, North-Holland, Amsterdam, 1973.

4. B. D. Calvert and A. G. Kartsatos, On the compactness of the nonlinear evolution operator in a Banach space, Bull. London Math. Soc. 19 (1987), 551-558.

5. M. G. Crandall and A. Pazy, Nonlinear evolution equations in Banach spaces, Israel J. Math. 11 (1972), 57-94.

6. L. C. Evans, Nonlinear evolution equations in an arbitrary Banach space, Israel J. Math. 26 (1977), 1-42.

7. S. Gutman, Compact perturbations of m-accretive operators in general Banach spaces, SIAM J. Math. Anal. 13 (1982), 789-800.

8. $\ldots$ Evolutions governed by m-accretive plus compact operators, Nonlinear Anal. TMA 7 (1983), 707-715.

9. A. G. Kartsatos, $A$ direct method for the existence of evolution operators associated with functional evolutions in general Banach spaces, Funkcial. Ekvac. 31 (1988), 89-102.

10. _ A compact evolution operator generated by a nonlinear time-dependent $m$-accretive operator in a Banach space, Math. Ann. (to appear).

11. A. G. Kartsatos and M. E. Parrott, The weak solution of a functional differential equation in a general Banach space, J. Differential Equations 75 (1988), 290-302. 
12. A. G. Kartsatos and K. Shin, Solvability of functional evolutions via compactness methods in general Banach spaces, Nonlinear Anal. TMA 21 (1993), 517-535.

13. V. Lakshmikantham and S. Leela, Nonlinear differential equations in abstract spaces, Pergamon Press, Oxford, 1981.

14. N. H. Pavel, Nonlinear evolution operators and semigroups, Lecture Notes in Math., vol. 1260, Springer-Verlag, Berlin, 1987.

15. N. Tanaka, Nonlinear nonautonomous differential equations, Publ. Res. Inst. Math. Sci. 647 (1988), 36-56.

16. $\ldots$, On the existence of solutions for functional evolution equations, Nonlinear Anal. TMA 12 (1988), 1087-1104.

17. I. I. Vrabie, Compactness methods for nonlinear evolutions, Longman Sci. Tech., Essex, 1987.

Department of Mathematics, University of South Florida, Tampa, Florida 33620-5700

E-mail address: hermes@gauss .math.usf .edu 